\title{
Cavitas Biosensors: Noninvasive Approaches to Blood Glucose Monitoring for Diabetes Mellitus
}

\author{
Kohji Mitsubayashi \\ Department of Biomedical Devices and Instrumentation, Institute of Biomaterials and Bioengineering, \\ Tokyo Medical and Dental University, 2-3-10 Kanda-Surugadai, Chiyoda, Tokyo 101-0062, Japan
}

(Received May 28, 2018; accepted July 2, 2018)

Keywords: biosensor, blood glucose, diabetes, cavitas, soft contact-lens, mouthguard, biocompatibility

Cavitas sensors that are attached to body cavities, such as the contact lens and mouthguard, have been developed and demonstrated. Being neither implanted nor worn, the cavitas sensors have many advantages for the noninvasive biosensing of chemicals in the permanent body fluid in human body cavities. A soft contact lens (SCL) biosensor for monitoring the sugar content in tears was fabricated using biocompatible polymer and bio-MEMS techniques. It performs realtime monitoring of tear sugar content, which reflects the level of blood glucose. In addition, a wireless mouthguard glucose sensor system for diabetes mellitus has also been developed by incorporating a small potentiostat and a Bluetooth 4.0 low-energy (LE) transmitter to a transparent mouthguard. In the near future, such self-detachable cavitas sensors will allow us to obtain biochemical information, thus improving the quality of life and living standards through daily medicine (health care and presymptomatic and preventive medicine).

\section{Introduction}

Biological measurement in the human body has been investigated in the medical and health care fields. In particular, many flexible and/or wearable sensors have been developed and commercialized in the world because of their prospects in the human monitoring of relevant parameters in health care, sports, and medical applications. ${ }^{(1,2)}$ The majority of existing sensor devices focus on the assessment of physical parameters. Unfortunately, the investigation of noninvasive chemical measurements has been delayed, but it is required for collecting biological information in the form of next-generation wearable devices. The noninvasive chemical technique would also affect the control of the physical condition in patients suffering from lifestyle-related diseases such as diabetes. Self-monitoring of blood glucose (SMBG) is traditionally an invasive method and is most commonly performed by "finger-prick testing" using a blood glucose meter for diabetic patients. However, compliance is often impaired owing to the discomfort, pain, and risk of infection, which may induce anxiety or fear. A noninvasive approach has been required for safe and continuous blood glucose assessment.

Since the 1990s, the author has focused on the use of body cavity fluids for noninvasive biosensing. ${ }^{(3,4)}$ The human body has many body cavities such as the cavitas oris (oral cavity), 
cavitas pharyngis, saccus lacrimalis, cavitas nasi, cavitas abdominalis, cavitas infraglotticum, and cavitas larynges. (Cavitas is the etymological origin of the word "cavity" in Latin.) Some cavitas sensors have been developed for application in human body cavities (cavitas oris, cavitas pharyngis, and conjunctival sac) for the noninvasive monitoring of biomedical information in the permanent body fluid in human body cavities. The cavitas sensors form a new category of detachable medical sensors between implantable and wearable sensors (Fig. 1). ${ }^{(5)}$

In this article, some cavitas biosensors for monitoring tear glucose in the conjunctival sac and saliva sugar in the oral cavity are focused on for noninvasive blood glucose analysis in diabetes mellitus. An update of various potential cavitas sensors and techniques and devices is provided, along with their advantages, challenges, and future trends.

\section{Soft Contact Lens (SCL) Biosensor for Tear Glucose Monitoring}

Noninvasive glucose monitoring does not measure blood sugar directly, but relies instead on the measurement of glucose levels in other biological fluids in human body cavities. ${ }^{(6)}$ With reference to continuous measurement, relationships between general physical conditions and chemical constituents of biological fluids such as tears, mucus, sweat, and saliva were reported. ${ }^{(7-11)}$ For example, a correlation between glucose concentration in tears and blood sugar level was reported. ${ }^{(12)}$ The tear sugar level changes with a short time delay (approx. $5 \mathrm{~min}$ ) in comparison with the blood glucose level. ${ }^{(13)}$

In 1995, authors developed a flexible glucose sensor using immobilized glucose oxidase (GOD) within a gold-coated, hydrophilic polytetrafluoroethylene membrane without employing harmful substances in a simple fabrication process. ${ }^{(14)}$ The sensor was calibrated against glucose solutions from 6.7 to $662 \mathrm{mg} / \mathrm{L}$, which is a range including the tear sugar level. A nonuniform gold layer deposited using a mesh mask was more effective for immobilizing the enzyme within the polyvinyl alcohol bearing styrylpyridinium group (PVA-SbQ) matrix, giving a device with high current output and excellent calibration characteristics. Ethanol sterilization had a negligible effect on output current and therefore is a suitable method for use in tear sugar sensing.

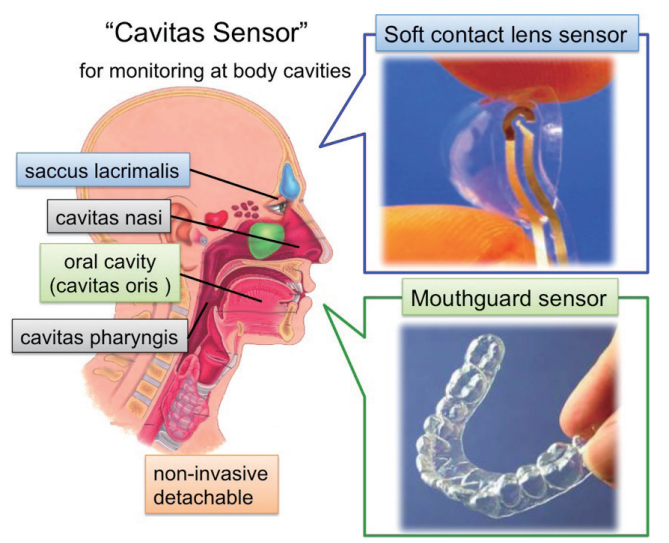

Fig. 1. (Color online) Concept image of cavitas sensors as detachable devices for noninvasive monitoring in body cavities. 
As the next step, another flexible biosensor was also developed for on-site tear sugar monitoring for physiological study. Such a biosensor was constructed by immobilizing GOD onto a flexible oxygen electrode (Pt working electrode and $\mathrm{Ag} / \mathrm{AgCl}$ counter/reference electrode), which was fabricated by soft-MEMS techniques on a gas-permeable polymer membrane. ${ }^{(15)}$ For bio-instrumentation, no adhesive agents were used for constructing the flexible biosensor. A linear relationship between glucose concentration and output current was obtained in the range of $0.025-1.475 \mathrm{mmol} / \mathrm{L}$, with a correlation coefficient of 0.998 . On the basis of the results of investigating the basic characteristics, the glucose sensor was applied to measure glucose in tear fluids on an eye site of a Japanese white rabbit. The temporal change in tear sugar level induced by the oral administration of glucose was monitored as a change in the current of the sensor fitted on the eye. In this investigation, the tear glucose level varied from 0.16 to $0.46 \mathrm{mmol} / \mathrm{L}$. Although there was a delay of several minutes compared with the blood sugar level, it is considered that noninvasive continuous glucose monitoring is possible using the flexible biosensor.

An SCL-type biosensor for the in situ monitoring of the tear sugar level was fabricated using biocompatible polymers for preclinical experiments (Fig. 2). ${ }^{(16)}$ The SCL biosensor was designed for the in situ monitoring of the tear sugar level. The most significant feature of the biosensor for tear fluid was that flexible and biocompatible polymers were used for all structural members. Poly(dimethylsiloxane) (PDMS) was used as the SCL body material. $\mathrm{Pt}$ and Ag electrodes were coated by sputtering with a thin Ti stencil without the use of any harmful chemicals. After forming the $\mathrm{Ag} / \mathrm{AgCl}$ layer, $\mathrm{GOD}$ was immobilized in the sensitive Pt region with another synthesized biocompatible polymer. The phospholipid polymer, the so-called MPC polymer, has a molecular configuration similar to that of a cell membrane. The novel biocompatible polymer PMEH [2-methacryloyloxyethyl phosphorylcholine (MPC) copolymerized with 2-ethylhexylmethacrylate (EHMA)] was also synthesized for enzyme immobilization (Fig. 3). A GOD and PMEH mixture in an ethanol solvent was coated for enzyme entrapping in the sensing region. The SCL sensor showed good flexibility and soft characteristics similar to those of a commercial available lens. This device retained its electrical performance after mechanical bending.
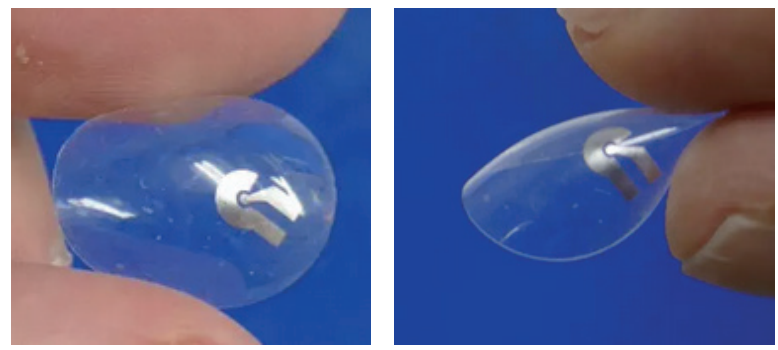

Fig. 2. (Color online) Photographs of SCL biosensor showing flexibility of not only the lens but also the coated electrodes.

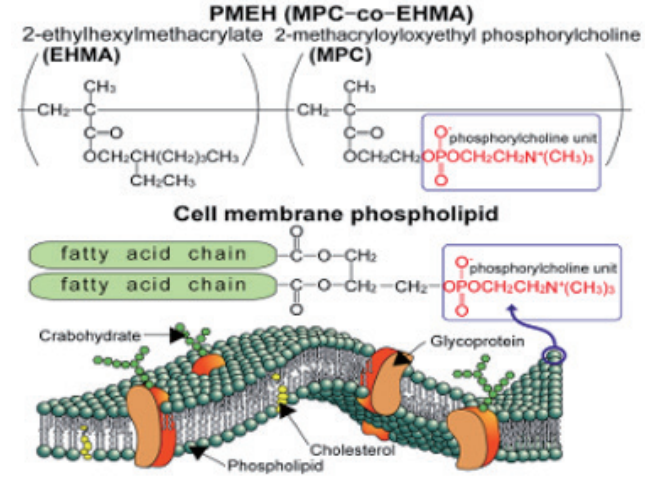

Fig. 3. (Color online) PMEH and phospholipid structure at cell membrane. 
The SCL biosensor indicated good relationship between the output current and the glucose concentration in the range of $0.03-5.0 \mathrm{mmol} / \mathrm{L}$, with a correlation coefficient of 0.999 . Also, the SCL biosensor was applied to a rabbit for tear glucose monitoring. The PDMS SCL was tentatively lead using a wired line in the preclinical experiment (Fig. 4). The wired SCL sensor enabled the successful real-time measurement of the tear glucose concentration, which is approximately one-tenth the blood glucose one. The basal tear glucose concentration was estimated to be $0.13 \mathrm{mmol} / \mathrm{L}$. The change in tear glucose concentration induced by a change in blood sugar level was also assessed by the oral glucose tolerance test (OGTT). As a result, the tear glucose level was found to increase with a delay of approximately $8 \mathrm{~min}$ from the blood sugar level (Fig. 4). This result demonstrated the relationship between the tear sugar and blood glucose levels with respect to the time delay. The result showed that the SCL biosensor is expected to provide further detailed information about the relationship between the dynamics of blood and tear glucose levels.

\section{Mouthguard Sensors in Oral Cavity}

Although there are numerous small glands distributed over the buccal mucosa, saliva is secreted mainly by the parotid and submaxillary and sublingual glands (Fig. 5). In general, saliva fluid includes many components such as amino acids, ions, proteins, sugar, nucleotides,
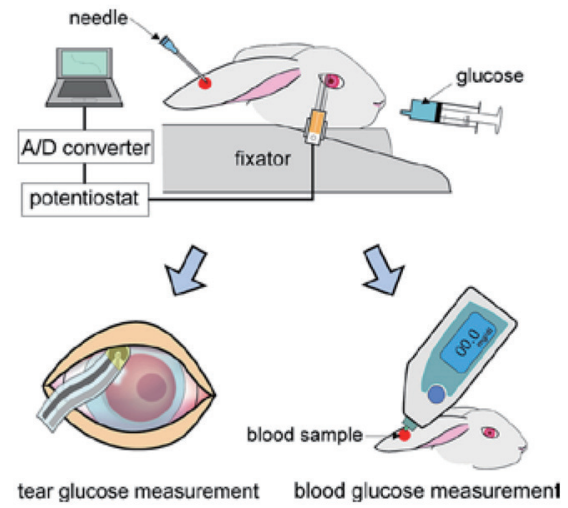

(a)

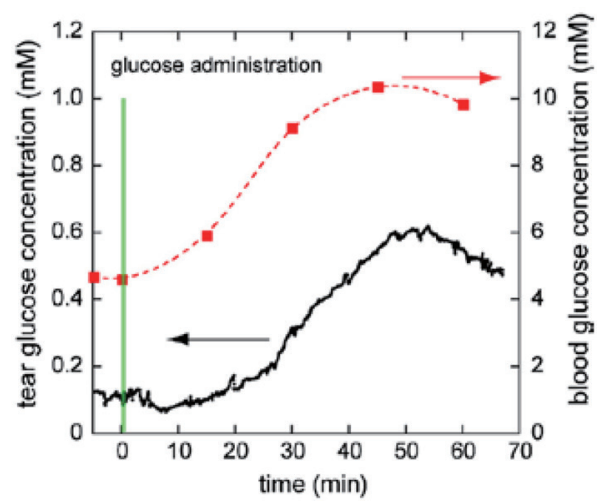

(b)

Fig. 4. (Color online) Schematic of OGTT. (a) The tear glucose concentration is measured with the SCL biosensor and the blood glucose concentration is measured with a commercially available test kit. (b) Temporal changes in tear and blood glucose levels after OGTT. ${ }^{(17)}$

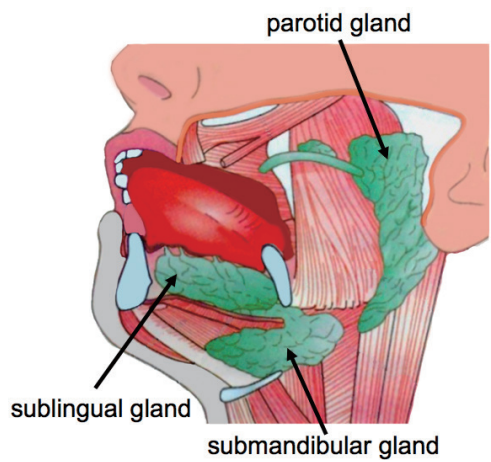

Fig. 5. (Color online) Schematic diagram of salivary glands. 
and microorganisms. Saliva is also a complex biofluid comprising numerous constituents permeating from blood via transcellular or paracellular paths. Saliva has attracted the attention of several researchers developing portable in vitro salivary diagnostic tools. ${ }^{(18)}$ Saliva is also a great diagnostic fluid and provides an alternative to direct blood analysis because of the permeation of blood constituents, without the need to pierce the skin for blood sampling.

We have developed a mouthguard (MG) biosensor for monitoring the saliva glucose level. $^{(5)}$ Saliva glucose concentrations approximately range from 20 to $200 \mu \mathrm{mol} / \mathrm{L}$ in normal and diabetic individuals, closely following circadian blood glucose fluctuations, ${ }^{(19)}$ and offer promising opportunities for noninvasive monitoring. ${ }^{(20)}$ Saliva and blood glucose levels correlate reasonably well in a sample of individuals. ${ }^{(21-24)}$ However, a much stronger correlation is observed within the same individual, enabling blood glucose concentrations to be estimated from saliva glucose measurements. ${ }^{(25)}$

A glucose sensor incorporating $\mathrm{Pt}$ and $\mathrm{Ag} / \mathrm{AgCl}$ electrodes with an enzyme membrane was developed and tested. The electrodes are formed on the surface of polyethylene terephthalate glycol (PETG) as the base MG material. Pt and Ag electrodes were formed on the PETG surface through sputtering. Each electrode consisted of a $0.20 \mathrm{~mm}^{2} \mathrm{Pt}$ working electrode and a 4.0 $\mathrm{mm}^{2} \mathrm{Ag} / \mathrm{AgCl}$ reference/counter electrode, both insulated with PDMS on a 0.5-mm-thick PETG layer. As the next step, the Pt working electrode was coated with a GOD membrane. 30 units of GOD were applied to the sensing region of the working electrode. In order to immobilize the enzyme, the biocompatible PMEH was used as the MG glucose sensor. $2.0 \mathrm{~mL}$ of $1.0 \mathrm{wt} \%$ PMEH solution was spread over the sensing region to form the PMEH overcoat. The glucose sensor and a wireless transmitter are seamlessly integrated into the MG biosensor. One small watch battery [1.5 V button battery (SR716SW)] was utilized as a power source between the MG materials inside the cheek (Fig. 6).

When investigating in vitro performance, the MG biosensor exhibits a robust relationship between output current and glucose concentration. In artificial saliva composed of salts and proteins, the glucose sensor is capable of highly sensitive detection over a glucose concentration range of 5-1000 $\mu \mathrm{mol} / \mathrm{L}$, which encompasses the range of glucose concentrations found in human saliva (Fig. 7). Artificial saliva containing various proteins was prepared from disodium hydrogen phosphate, anhydrous calcium chloride, potassium chloride, sodium chloride, urea,

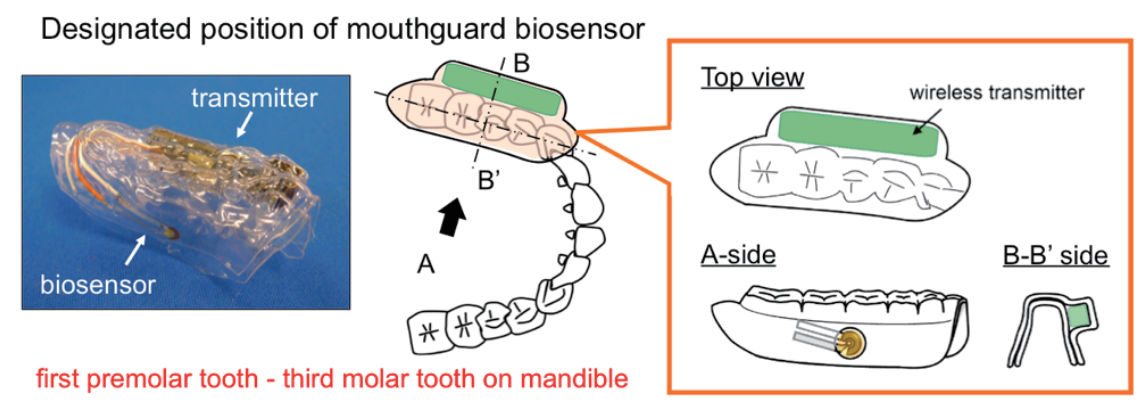

Fig. 6. (Color online) Schematic image of the mouthguard biosensor custom-fit to the patient's teeth. The device consisted of a glucose sensor and a wireless transmitter incorporating a potentiostat for stable glucose measurement. The wireless transmitter was neatly encased in PETG. 


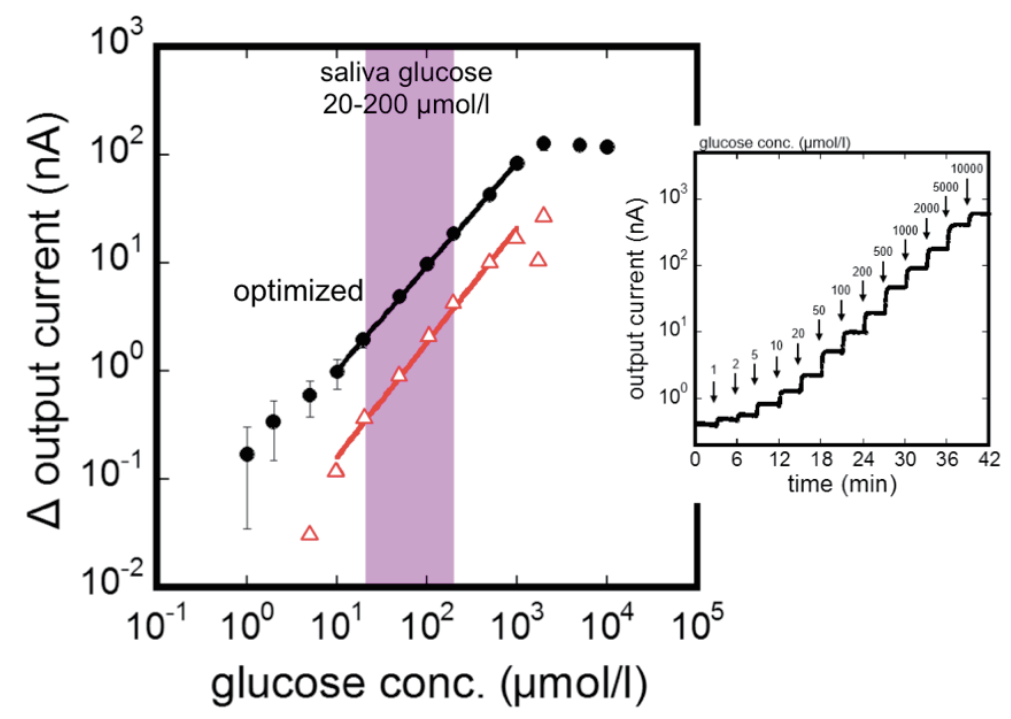

Fig. 7. (Color online) Calibration curves of the optimized glucose sensor on PETG. The calibration range was $10-1000 \mu \mathrm{mol} / \mathrm{L}$, which encompassed the physiological saliva glucose range in humans $(20-200 \mu \mathrm{mol} / \mathrm{L})$. The output current of the glucose sensor without a PMEH overcoat was substantially decreased because of proteins in artificial saliva adhering to the electrode surface.

and type II mucin from porcine stomach, in accordance with a protocol reported by Fusayama et al. ${ }^{(26)}$ The ability of the sensor and wireless communication module to monitor saliva glucose was demonstrated in a phantom jaw imitating the structure of the human oral cavity. Stable and long-term real-time monitoring (exceeding $5 \mathrm{~h}$ ) with the wireless system was achieved. The MG biosensor will be useful as a novel tool for real-time noninvasive saliva glucose monitoring for better management of dental patients. In addition, the selectivity of the MG sensor was evaluated by comparing output currents in response to solutions with $100 \mu \mathrm{mol} / \mathrm{L}$ glucose, galactose, fructose, mannitol, sorbitol, and xylitol after $180 \mathrm{~s}$. The glucose sensor was shown to be highly selective for glucose on the basis of the substrate specificity of GOD. Fructose, mannitol, sorbitol, and xylitol included in some foods were not detected, producing a negligible output current with less than $0.05 \%$ of the magnitude of the output current produced by glucose. Galactose was detected to a minimal extent, producing an output current at $0.265 \%$ of the magnitude of the output current produced by glucose.

In our laboratory, an invisible MG biosensor using thinner and transparent dental materials integrated with a small Bluetooth low-energy (BLE) wireless module for the real-time monitoring of saliva glucose concentration has also been developed. The novel MG biosensor was integrated with a glucose sensor and a BLE wireless measurement system. The electrodes were formed on the mouthguard surface, which was made of transparent PETG. Stable and long-term monitoring (more than $10 \mathrm{~h}$ ) using a telemetry system was established. The thinner MG biosensor would be useful for a real-time, noninvasive and automatic method of health care management for a patient in the dental field. Also, this invisible smart mouth gear (Fig. 8) would be useful for future daily medicine. 


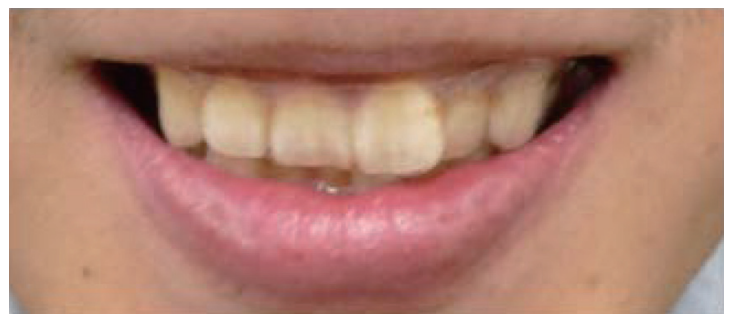

(a)

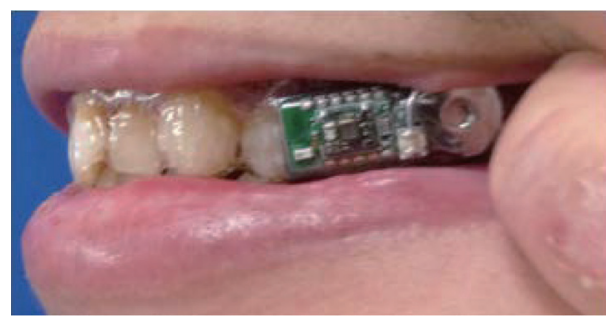

(b)

Fig. 8. (Color online) Photographs [(a) front and (b) side views] of invisible smart mouth gear for monitoring salivary glucose concentration. The wireless mouthguard biosensor is custom-fit to the patient's teeth. The wireless transmitter was neatly encased in PETG MG material.

\section{Conclusions}

Both the SCL-and MG-type biosensors of cavitas devices were developed with biocompatible polymers and bio-MEMS techniques. The SCL-type biosensor showed good relationship between the output current and the glucose concentration in a range of $0.03-5.0 \mathrm{mmol} / \mathrm{L}$, with a correlation coefficient of 0.999 . The wired SCL sensor enabled the successful assessment of the change in tear glucose concentration induced by a change in blood sugar level assessed by the OGTT, demonstrating the relationship between the glucose concentrations in tears and blood with a time delay.

The wireless MG biosensor integrated with a small BLE transmitter module was capable of highly sensitive detection over a glucose concentration range of 5-1000 $\mu \mathrm{mol} / \mathrm{L}$, which encompasses the range of glucose concentrations found in human saliva. A thinner and invisible mouthguard biosensor would be useful for a real-time, noninvasive and automatic method of health care management for a patient in the dental field.

Human secretions such as tears, saliva, sweat, and body gas will provide important bioinformation about the health and well-being of an individual. In the near future, many types of cavitas sensors will be developed and commercialized for managing the aging society in the world. Daily medical care (health care and presymptomatic and preventive medicine) with cavitas and wearable sensors is necessary to improve the quality of life in view of the aging of society and the rapid changes in living environments.

\section{References}

1 E. Ghafar-Zadeh: Sensors 15 (2015) 3236.

2 S. R. Corrie, J. W. Coffey, J. Islam, K. A. Markey, and M. A. F. Kendall: Analyst 140 (2015) 4350.

3 K. Mitsubayashi, K. Yokoyama, T. Takeuchi, E. Tamiya, and I. Karube: Anal. Chem. 65 (1993) 3586.

4 K. Mitsubayashi, K. Ogasawara, K. Yokoyama, T. Takeuchi, T. Tsuru, and I. Karube: Technol. Health Care 3 (1995) 117.

5 T. Arakawa, Y. Kuroki, H. Nitta, P. Chouhan, K. Toma, S. Sawada, S. Takeuchi, T. Sekita, K. Akiyoshi, S. Minakuchi, and K. Mitsubayashi: Biosens. Bioelectron. 84 (2016) 106.

6 G. S. Wilson and R. Gifford: Biosens. Bioelectron. 20 (2005) 2388.

7 K. M. Daum and R. M. Hill: Invest. Ophthalmol. Visual Sci. 22 (1982) 509. 
8 S. F. P Man, G. K. Adams, and D. F. Proctor: J. Appl. Physiol.: Respir. Environ. Exercise Physiol. 46 (1979) 205.

9 K. Mitsubayashi, M. Suzuki, E. Tamiya, and I. Karube: Anal. Chim. Acta 289 (1994) 27.

10 A. Romano and F. Rolant: Metab. Pediatr. Syst. Ophthalmol. 11 (1988) 78.

11 T. R. Stolwijk, J. A. van Best, H. H. P. J. Lemkes, R. J. W. de Keizer, and J. A. Oosterhuis: Int. Ophthalmol. 15 (1991) 377.

12 D. K. Sen and G. S. Sarin: Br. J. Ophthalmol. 64 (1980) 693.

13 W. F. March, F. E. Smith, P. Herbrechtsmeier, A. Domschke, and C. Morris: Diabetes 50 (2001) A125.

14 K. Mitsubayashi, J. M. Dicks, K. Yokoyama, T. Tamiya, and I. Karube: Electroanalysis 7 (1995) 83.

15 S. Iguchi, H. Kudo, T. Saito, M. Ogawa, H. Saito, K. Otsuka, A. Funakubo, and K. Mitsubayashi: Biomed. Microdevices 9 (2007) 603.

16 M. X. Chu, H. Kudo, T. Shirai, K. Miyajima, H. Saito, N. Morimoto, K. Yano, Y. Iwasaki, K. Akiyoshi, and K. Mitsubayashi: Biomed. Microdevices 11 (2009) 837.

17 M. X. Chu, K. Miyajima, D. Takahashi, T. Arakawa, K. Sano, S. Sawada, H. Kudo, Y. Iwasaki, K. Akiyoshi, M. Mochizuki, and K. Mitsubayashi: Talanta 83 (2011) 960.

18 A. Vasudeva, A. Kaushik, Y. Tomizawa, N. Norena, and S. Bhansali: Sens. Actuators, B 182 (2013) 139.

19 M. Yamaguchi, M. Mitsumori, and Y. Kano: IEEE Eng. Med. Biol. Mag. 17 (1998) 59.

20 P. Mascarenhas, B. Fatela, and I. Barahona: PLoS One 9 (2014) e101706.

21 C. Jurysta, N. Bulur, B. Oguzhan, I. Satman, T. M. Yilmaz, W. J. Malaisse, and A. Sener: J. Biomed. Biotechnol. 2009 (2009) 430426.

22 P. Abikshyeet, V. Ramesh, and N. Oza: Onco Targets Ther. 5 (2012) 149.

23 C. Liu, Y. Sheng, Y. Sun, J. Feng, S. Wang, J. Zhang, J. Xu, and D. Jiang: Biosens. Bioelectron. 70 (2015) 455.

24 A. Soni and S. K. Jha: Biosens. Bioelectron. 67 (2015) 763.

25 W. Zhang, Y. Du, and M. L. Wang: Sens. Bio-Sens. Res. 4 (2015) 96.

26 T. Fusayama, T. Katayori, and S. Nomoto: J. Dent. Res. 42 (1963) 1183. 